${ }^{4}$ Department of Rheumatology, Third Affiliated Hospital of Soochow University, Changzhou; ${ }^{5}$ Department of Rheumatology, Liangyungang First People's Hospital, Liangyungang; ${ }^{6} 8$ Department of Rheumatology, Subei People's Hospital of Jiangsu province, Yangzhou; ${ }^{7}$ Department of Rheumatology, Wuxi People's Hospital, Wuxi, China

Background: Nowadays the importance of antimalarials, especially hydroxychloroquine (HCQ) and chloroquine (CQ), in treatment of systemic lupus erythematosus (SLE) has been demonstrated. However, few have examined the efficacy of $\mathrm{HCQ}$ and $C Q$ on eastern Chinese SLE patients.

Methods: The analysis is based on 1372 patients who were enrolled in a retrospective study of 26 centers from January 1st, 1999 through December $31 \mathrm{st}, 2009$, during which time is their first hospitalization. Baseline and follow-up clinical, laboratory and therapeutic data and survival status before April 30th, 2015 were recorded. Statistical analysis consist of Chi-square test, $t$-test, Kaplan-Meier curves and logrank test.

Results: Compared with 562 patients without $\mathrm{HCQ}$ or $\mathrm{CQ}$ treatment, the hazard ratio (HR) of deaths in 810 patients taking those was reduced (HR $0.52,95 \%$ $\mathrm{Cl} 0.38-0.70, p<0.001) .376$ of these 1372 patients experienced their second hospitalization, during which treating group (165 of 376) showed lower blood level of total cholesterol (TG), compared to control group (4.47 (0.13) vs $5.03(0.21)$, $p=0.027$ ), while no statistical difference of TG exists between the two groups' first hospitalization $(p>0.05)$. Other metabolic data, such as systolic and diastolic blood pressure, fasting blood sugar, Triglyceride and uric acid were similar between the two groups in two times of hospitalization. On second inpatient visit, disease activity (SLEDAI, blood sedimentation rate, complement) and organ involvements (SLICC) of those who took antimalarials and no users have no significant differences.

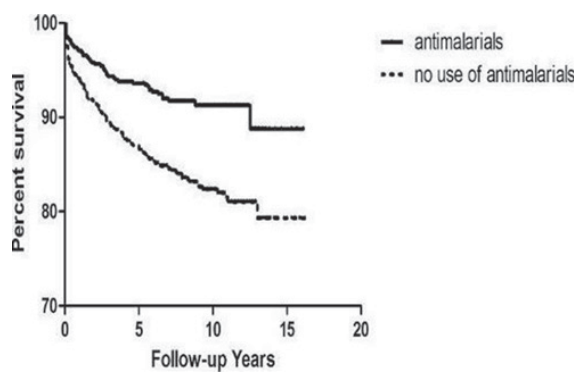

Conclusions: Use of $\mathrm{HCQ}$ or $\mathrm{CQ}$ lower the risk of mortality and TG levels of eastern Chinese SLE patients.

Disclosure of Interest: None declared

DOI: 10.1136/annrheumdis-2017-eular.4208

\section{AB0448 USE OF DENOSUMAB IN PATIENTS WITH SYSTEMIC LUPUS ERYTHEMATOSUS: A REAL LIFE MULTI-CENTRIC EXPERIENCE}

F. Crisafulli ${ }^{1}$, M. Fredi ${ }^{2}$, A. Bortoluzzi ${ }^{3}$, I. Cavazzana ${ }^{2}$, F. Furini $^{3}$, M. Govoni ${ }^{3}$, A. Tincani ${ }^{1}$, F. Franceschini ${ }^{2} .{ }^{1}$ Rheumatology Unit and University of Brescia; ${ }^{2}$ Rheumatology Unit, Brescia; ${ }^{3}$ Department of Medical Sciences, Rheumatology Unit and University of Ferrara, Ferrara, Italy

Background: The occurrence of osteoporosis (OP) and fragility fractures (FF) is a frequent comorbidity in patients with systemic lupus erythematosus (SLE), mainly due to the concomitant presence of many risks factors for secondary osteoporosis (drugs, gender, concomitant diseases). The use of corticosteroids (CS) increases the risk of osteoporosis induced by corticosteroid (GIOP) and FF [1]. Denosumab is monoclonal antibody that binds to RANKL, inhibiting osteoclast formation and activation, used for both male and women OP to prevent FFx [2,3]. Previous RCT [2] reported a numerically higher serious adverse events of infections in the denosumab users. In particular severe skin infections were significantly more frequent [4]. The possible increase in infection risk might be a concern in subjects treated with CS. The use of denosumab in SLE with GIOP patients is recently reported in 17 cases within a RCT [5]. Anyway, no data regarding the tolerability and disease activity during therapy were reported

Objectives: Our aim was to analyze the prevalence, the tolerability, and relevant changes in disease activity or damage in a cohort of SLE patients treated with denosumab

Methods: among all SLE patients currently followed in two referral Center we selected the ones that received at least one dose of denosumab. Clinical, serological manifestations, concomitant diseases and therapies were collected from clinical charts. Damage index (SDI), activity index (SLEDAI-2K) were calculated when denosumab was introduced (first cycle) and at the last evaluation (last cycle). For statistical analysis Chi-squared test or Fisher exact test was used Results: Among 793 patients in our cohorts, 21 (2.6\%) were treated with denosumab. Demographic data reported a female prevalence (19 cases, $90 \%$ ), mean age at onset of $38 \pm 12$ years, mean duration of disease of $28 \pm 10 y e a r s$ and mean follow-up of $21 \pm 7.8$ years; most frequent manifestations were arthritis $(90 \%)$, cutaneous $(67 \%)$ and neurological $(67 \%)$. All patients were still treated with CS (mean duration of $25 \pm 6.8$ years) with a daily dose of $8.5 \pm 3.5 \mathrm{mg} /$ day. Other risk factors for OP were present in the majority of them: drugs (anticoagulants

\begin{tabular}{|c|c|c|c|}
\hline Efficacyladverse events & First cycle ( $N=21$ pts) & Last cycle ( $\mathrm{N}=21 \mathrm{pts}$ ) & $P$ value \\
\hline New fracture & Not applicable & $0(0 \%)$ & na \\
\hline Mean dose of $\mathrm{GC}, \mathrm{mg} /$ day (mean $\pm \mathrm{DS}$ ) & $6.9 \pm 3.4$ & $5.8 \pm 3.1$ & ns \\
\hline T score hip* & $-2.6 \pm 1.4$ & $-2 \pm 1$ & ns \\
\hline T score lumbar spine * & $-2.61 \pm 1.1$ & $-2.3 \pm 0.8$ & ns \\
\hline Hypovitaminosis D ** & $15(71 \%)$ & $13(62 \%)$ & ns \\
\hline SLEDAI-2k (mean \pm DS) & $3.5 \pm 3.3$ & $2.36 \pm 2.65$ & ns \\
\hline SDI (mean $\pm D S)$ & $4 \pm 1.18$ & $4.1 \pm 1.21$ & ns \\
\hline Any adverse event & 1 & 1 & ns \\
\hline Infective episodes & 1 (not requiring antibiotic) & $\begin{array}{l}1 \text { (not requiring antibi- } \\
\text { otic) }\end{array}$ & \\
\hline Skin infection & 0 & 0 & ns \\
\hline Flare SLE & 0 & 1 (arthritis) & ns \\
\hline Therapy withdrawal & na & 0 & ns \\
\hline
\end{tabular}

in 6pts, cyclosporin in 3 and antiepileptic in 2), chronic kidney disease (CKD) (4pts), hypovitaminosis D (15pts), anorexia, celiac disease and hemochromatosis (1pt, each). Indication for denosumab was OP with FFx in 17 cases: denosumab was used for a new FF during biphosphonates or after teriparatide. In the other 4 the indication was primary prevention with contraindications at the use of biphosphonates for concomitant severe CKD. Mean duration therapy was 4 cycles (range 1-8): data regarding disease activity, damage and adverse events are reported in table 1 . No new FFx developed in any of the included patients at the last evaluation available.

Conclusions: In our cohorts denosumab is still used in few selected patients. However it could be considered as a valid option in GIOP patients because it was globally well tolerated and in our cohort it's efficacy in the prevention of new fragility fractures was confirmed

References:

[1] Gudbjornsson, et al. Ann Rheum Dis 2002;61,32-36.

[2] Cummings SR et al. N Eng J Med 2009; 61:756-65.

[3] Austin M et al. J Bone Miner Res 2012;27:687-93.

[4] Watts NB, Osteoporos Int 2012;23:327-37.

[5] Mok CC, et al. Bone 2015;75:222-8.

Disclosure of Interest: None declared

DOI: 10.1136/annrheumdis-2017-eular.2740

\section{AB0449 OFF LABEL OF BIOLOLOGICS IN CONNECTIVE TISSUE DISEASES. A SINGLE CENTER EXPERIENCE}

G. Orsolini ${ }^{1}$, C. Crowson ${ }^{2}$, O. Viapiana ${ }^{1}$, M. Rossini ${ }^{1}$, K.G. Moder ${ }^{2}$

${ }^{1}$ Rheumatology, University of Verona, Verona, Italy; ${ }^{2}$ Rheumatology, Mayo Clinic, Rochester, United States

Background: Connective tissue diseases (CTDs) are a broad spectrum of autoimmune conditions including different entities such as systemic sclerosis (SSc), Sjogren syndrome (SSj), systemic lupus erythematosus (SLE) and autoimmune myositis. There are many patients that do not meet the classification or diagnostic criteria and they fall under the diagnosis of Undifferentiated connective tissue disease (UCTD). Treatment of refractory forms of these could be challenging and clinicians sometimes are forced to try off label drugs such as biologics on the basis of scarce literature support and the experience on different rheumatic disease.

Objectives: To evaluate duration of biologic drug administered off label for the treatment of CTD and investigate variables possibly associated to drug suspension.

Methods: We used ACE program to search among Mayo Clinic clinical records all the patients that had in their records the words "undifferentiated connective tissue disease" or "lupus" or "myositis" or "Sjogren" or "systemic sclerosis" or "scleroderma"(and acronyms) AND "infliximab" or "etanercept" or "golimumab" or "certolizumab" or "tocilizumab" or "abatacept" or "adalimumab" (and their brand names).

All records were checked for definite diagnosis and patients with uncertain ones where excluded. Also medications were checked, patients without any information about treatments where excluded. All the records of the selected patients were used to collect information about the off label biologic treatment but also clinical, serological and demographic variables.

Results: We collected data on 122 patients with connective tissue diseases, some of them had other concomitant autoimmune diseases with indication for biologic treatment (e.g. rheumatoid arthritis, etc). We analyze the group with some CTD alone $(n=72)$ considering SLE $(n=18-25 \%)$, inflammatory myositis $(n=22$ $-31 \%)$ and UCTD ( $n=32-44 \%)$. In this group the first biologic was etanercept in $55 \%$, adalimumab in $18 \%$, infliximab in $17 \%$, golimumab in $1 \%$ and abatacept in $8 \%$. The $22 \%$ tried also a second biologic and $4 \%$ a third. We consider for analysis the first treatment.

Mean treatment duration was $0.8( \pm 1.1)$ years. In our population $12,5 \%$ experienced a flare of CTD, $12,5 \%$ had infections, $18 \%$ had allergic reactions (of any type). The $44 \%$ experienced primary failure, $11 \%$ loss of efficacy, $31 \%$ had minor adverse events, $14 \%$ major ones (possible more than one reason for interruption).

Analysis showed no definite factors correlated with treatment duration or failure or adverse events, No difference due to type of CTD.

Conclusions: The study is retrospective and this limits the conclusions to be taken from it, however is one of largest population of off label treatments in CTDs 